\title{
UNA APROXIMACIÓN AL SEGUIMIENTO A EGRESADOS DE EDUCACIÓN NO FORMAL EN EL DCA-FACES
}

\author{
Ana-Lucía Ulloa-Cadalso ${ }^{1, *}$, Alex Matamoros-Castro ${ }^{1,+}$ \\ ${ }^{1}$ Departamento de Ciencia Aeronáuticas, Facultad de Ciencias Espaciales, UNAH \\ Recibido: $01 /$ julio/2019 \\ Aceptado: 06/julio/2019 \\ DOI: https://doi.org/10.5377/ce.v12i2.10288
}

\begin{abstract}
RESUMEN
La presente investigación analiza la formación impartida por el Departamento de Ciencias Aeronáuticas (DCA) de la UNAH para facilitar el seguimiento a egresados. Para lograr este objetivo se hizo una caracterización de los egresados de los diplomados aeronáuticos impartidos por el DCA. Se aplicaron dos instrumentos de consulta: una encuesta y una entrevista; los consultados cumplían con los criterios de: estar activos en el campo para el cual fueron formados, ser profesionales de los que el DCA contaba con sus contactos y mostraron disponibilidad a ser consultados. De los resultados destacan, el uso intensivo de los conocimientos adquiridos y la percepción de los beneficios de la formación recibida; además, los entrevistados mencionaron aportes de los diplomados como la comprensión de los perfiles que interactúan en la aeronáutica, la inducción a la investigación, el proveer una visión global de las Ciencias Aeronáuticas y la aplicación de conocimientos para la mejora de procesos. En el apartado de Discusión se ofrecen entre otras reflexiones las variables que debería incluir un banco de datos de egresados del DCA. Se concluye que los conocimientos y habilidades adquiridos o estimulados por los dos diplomados cursados en el DCA, han mejorado el desempeño de los técnicos y profesionales egresados, los cuales se encuentran prestando sus servicios en un abanico amplio de empresas e instituciones aeronáuticas, mismas que constituyen una fuente importante de información para el establecimiento de una oferta académica del DCA, acorde con las necesidades reales de formación de la comunidad aeronáutica de Honduras.
\end{abstract}

Palabras clave: Seguimiento a egresados, banco de datos, formación.

\begin{abstract}
This research analyzes the training given by the Department of Aeronautical Sciences (DCA) of UNAH to facilitate the monitoring of graduates. To achieve this objective, a characterization of the graduates of the aeronautical diplomas taught by the DCA was made. Two instruments of consultation were applied: survey and interview, the respondents met the criteria of: being active in the field for which they were trained, being professionals of whom the DCA had their contacts and showed willingness to be consulted. Of the results, the intensive use of the acquired knowledge and the perception of the benefits of the training received stand out; In addition, the interviewees mentioned the contributions of the graduates such
\end{abstract}

*ana.ulloa@unah.edu.hn

†amatamoros@unah.edu.hn 
as the understanding of the profiles that interact in aeronautics, the induction of research, providing a global vision of aeronautical sciences and the application of knowledge to improve processes. In the Discussion section, among other reflections, the variables that a database of DCA graduates should include are offered. It is concluded that the knowledge and skills acquired or stimulated by the two graduates studied at the DCA have improved the performance of the graduated technicians and professionals, who are providing their services in a wide range of aeronautical companies and institutions, which constitute An important source of information for the establishment of an academic offer of the DCA, in accordance with the real training needs of the Honduran aeronautical community.

Keywords: Follow-up to graduates, data bank, training.

\section{Introducción}

El seguimiento a egresados es para la Universidad Nacional Autónoma de Honduras una función vital, de ahí que se haya instituido un Departamento dedicado a esta finalidad; entre las funciones de dicho Departamento están las de revisar la pertinencia de la formación de los egresados y la definición de los requerimientos de actualización (UNAH, 2008). La Dirección de Vinculación Universidad Sociedad cuenta con un sistema de indicadores de seguimiento para el monitoreo de estos y otros aspectos de seguimiento a graduados, a la espera de ser implementado (DVUS, 2016).

Por su parte el Departamento de Ciencias Aeronáuticas de la Facultad de Ciencias Espaciales de la UNAH, a la fecha ha formado un número importante de egresados de distintos procesos de educación no formal (ver tabla 1) a los que debería estar dando seguimiento de forma sistemática.

Tabla 1: Descripción de diplomados impartidos según número de ediciones y número de egresados. Proyectos de vinculación del DCA período 2015-2018

\begin{tabular}{llcc}
\hline $\mathbf{N}^{\circ}$. & Proceso formativo & Ediciones & Egresados \\
\hline 1 & $\begin{array}{l}\text { Diplomado en Gestión de Sistemas Aeroportuarios (DCA, 2015) } \\
2\end{array}$ & $\begin{array}{l}\text { Diplomado en Gestión de la Aeronavegabilidad y Mantenimiento Ae- } \\
\text { ronáutico (DCA, 2018b) }\end{array}$ & 31 \\
3 & Seminario de Navegación Aérea, Tendencias Internacionales y su In- & 1 & 25 \\
& fluencia en la Región Centroamericana (DCA, 2018c) & 38 \\
4 & Seminario de Mantenimiento Aeronáutico e Ingeniería & 2 & 24 \\
5 & Curso Formación de Instructores Técnicos Aeronáuticos (DCA, 2018a) & 1 & 18 \\
6 & Curso Aeródromos Municipales & 2 & 47 \\
7 & Seminario Introducción a Sistemas Aéreos no Tripulados de Opera- & 1 & 18 \\
& ción Remota (RPAS) & & \\
8 & Seminario Certificación de Aeródromo & 1 & 13 \\
9 & Curso Peso y Balance de Aeronaves & 3 & 47 \\
10 & Seminario Certificación de Centros de Instrucción Aeronáutica RAC & 1 & 3 \\
& 141 & & \\
11 & Ciclo de conferencias & 3 & 152 \\
\hline Totales & & 446 \\
\hline
\end{tabular}

Como se muestra en la tabla anterior la oferta formativa del DCA es relativamente amplia y variada. Sin embargo, para la presente investigación se tomaron los datos de egresados de los diplomados, puesto 
que son procesos formativos que cuentan con la mayoría de elementos incluidos en la educación formal, a saber: matrícula formal, combinación de actividades presenciales y virtuales, actividades prácticas de campo y talleres, asesoría a los estudiantes, tareas para desarrollar fuera del aula de clases, investigaciones, presentaciones por parte de los estudiantes como requisito de egreso de investigaciones y proyectos de mejoramiento de procesos para aplicar los conocimientos adquiridos.

Un referente importante para el presente trabajo de investigación es la gestión del conocimiento entendido como el uso de los conocimientos institucionales e individuales para el mejor desempeño posible de la institución, organización o empresa poseedora de estos conocimientos (del Moral Bueno et al., 2007). Al establecer una dinámica de gestión de los conocimientos difundidos y empleados en sus procesos formativos el DCA, estará colaborando con la comunidad aeronáutica nacional hondureña, proveyéndole retroalimentación sobre las necesidades de formación de sus empleados. Esta iniciativa de colaboración está en consonancia con el Modelo Educativo de la UNAH que destaca en el estudiante egresado la capacidad para "resolver problemas cotidianos en espacios concretos" (UNAH, 2009) y, con las Normas Académicas que establecen como tema constante de investigación el seguimiento a graduados (Art. 267), los cuales deben contar con conocimientos actualizados sobre el campo en el cual se desempeñan; de hecho, "Toda carrera de grado y posgrado con la participación de los docentes de la Escuela o el Departamento a la cual la carrera está adscrita, deberá periódicamente (por lo menos cada cinco años ), determinar la pertinencia de la formación recibida por los graduados en relación con las demandas de la sociedad, y del desarrollo humano sostenible" (UNAH, 2015).

\section{Metodología}

Los datos recogidos para la presente investigación corresponden al período 2015-2018 y están referidos a actividades realizadas en las Ciudades de Tegucigalpa y San Pedro Sula. Asumiendo la condición explicativa de la investigación propuesta, se enlistan algunas de las características que poseen los exalumnos de programas de formación ofrecidos por el DCA, así como las aportaciones de los mismos a las instituciones y/o empresas donde laboran. Dado que los aspectos seleccionados se revisaron una sola vez, se considera una investigación transversal. Sin embargo, con los aportes de esta investigación se espera establecer un conjunto de variables que servirán de base para elaborar un banco de datos que permita desarrollar estudios longitudinales de seguimiento a egresados de la oferta académica del DCA.

Se consultó como fuente primaria a los exalumnos egresados del Diplomado en Gestión de Sistemas Aeronáuticos y a los del Diplomado en Gestión de la Aeronavegabilidad y el Mantenimiento Aeronáutico; cada uno de estos diplomados ha desarrollado tres ediciones de las cuales provienen 76 egresados. Las fuentes secundarias fueron los perfiles de los diplomados antes mencionados, las estadísticas generadas por el DCA de exalumnos egresados de sus programas educativos y el Modelo Educativo de la UNAH.

Luego de hacer una revisión bibliográfica se aplicaron como instrumentos de consulta, una entrevista en profundidad con el Encargado de seguimiento a graduados de la Dirección de vinculación Universidad Sociedad de la UNAH, una encuesta a egresados de diplomados y un cuestionario a un grupo de egresados seleccionado aplicando los criterios de estar activos en el campo para el cual fueron formados, ser profesionales de los que el DCA contaba con sus contactos y mostrar disponibilidad a ser consultados. Los instrumentos aplicados se orientaron a examinar aspectos como las habilidades desarrolladas, el valor de uso dado a los contenidos de la formación recibida, la preferencia de los entrevistados por la formación recibida con el DCA. 


\section{Resultados}

1. Análisis de la encuesta

1.1 Vigencia del conocimiento. La mayoría de los encuestados recibieron su diplomado entre febrero de 2014 y junio de 2015 (51.5\%), entre el último mes mencionado y el presente (agosto de 2018) han transcurrido 3 años, no obstante, se considera que los conocimientos adquiridos son todavía vigentes puesto que los contenidos tratados tienen, una velocidad moderada de cambio, se trató de Anexos al Convenio de Chicago, Regulaciones de Aviación Civil, Estructuras de manuales, legislación aeronáutica, estructuras tipo de autoridades aeronáuticas; así como estructuras de Directivas de Aeronavegabilidad o AD notes, certificados tipo y otros documentos similares (ver gráfico de la figura 1).

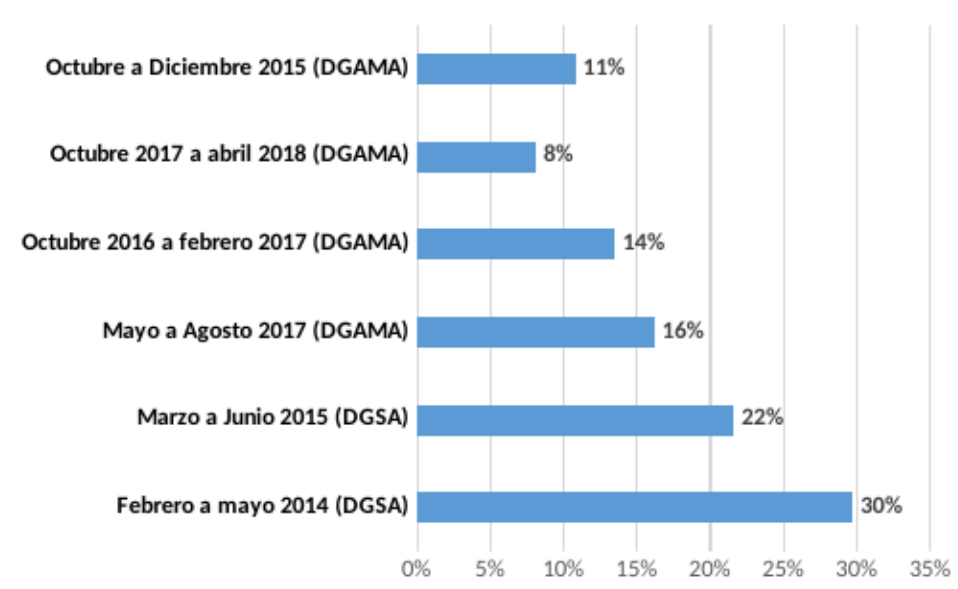

Figura 1: Fecha en que se cursó el Diplomado. Fuente: elaboración propia.

1.2 Áreas fortalecidas. En el caso de DGSA se reportaron fortalezas en: Aeropuertos, operaciones, seguridad $(72.7 \%)$ y en el caso del DGAMA: Mantenimiento de la Aeronavegabilidad y Operadores aéreos, cumplimiento de regulaciones; ambos ítems obtuvieron 10 de las doce respuestas hechas a la pregunta sobre los temas en los que se consideraban mejor formados (ver gráficos de las figuras 2 y 3).

1.3 Aplicación del conocimiento. Al momento de aplicar la encuesta los egresados tenían presencia en 15 instituciones o empresas del rubro aeronáutico en donde un $81.8 \%$ de los encuestados dijo estar aplicando los conocimientos adquiridos. Este hecho convierte a los consultados en un buen referente para que el DCA considere sus criterios para revisar la pertinencia de los conocimientos utilizados y por utilizar en sus propuestas formativas (ver gráfico de la figura 4). Con el resto de la población, el DCA debería profundizar en las razones por las cuales no se están aplicando los conocimientos adquiridos, las respuestas que se obtengan en este caso deberían orientar nuevas propuestas formativas.

1.4 Beneficios de la formación. La gran mayoría de los consultados (97\%) dijo que la formación recibida en los diplomados fue beneficiosa a nivel personal; de hecho, en un $61.9 \%$ de los casos el diplomado recibido fue un elemento que ayudó a conseguir un empleo dentro del rubro aeronáutico (ver gráfico de la figura 5). 


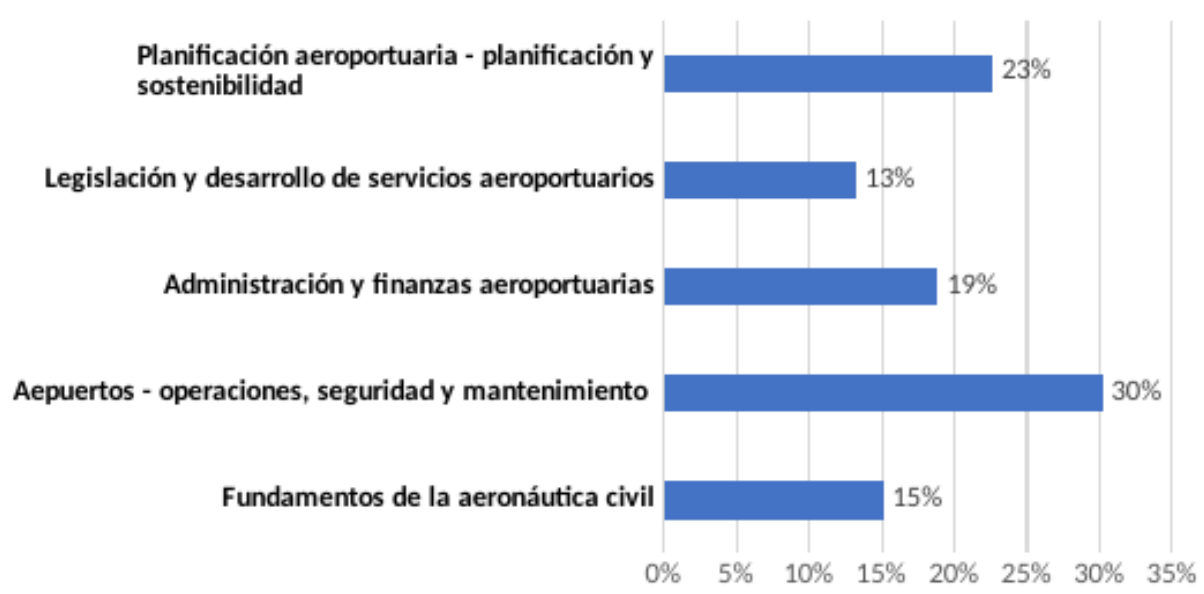

Figura 2: Temas en los que se percibe mayor fortaleza - DGSA. Fuente: Elaboración propia.



Figura 3: Temas en los que se percibe mayor fortaleza - DGMA. Fuente: Elaboración propia.

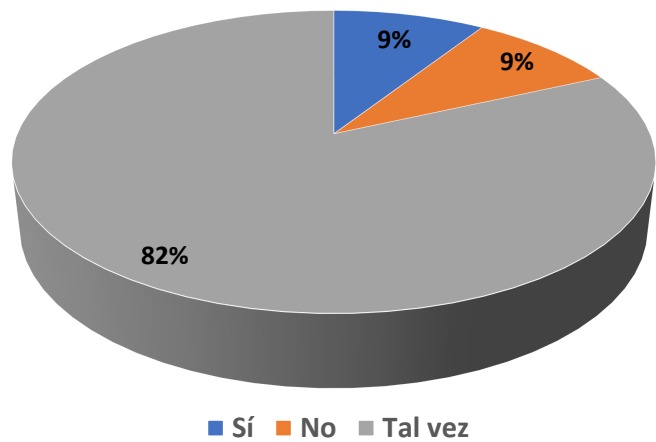

Figura 4: Aplicación de los conocimientos adquiridos. 


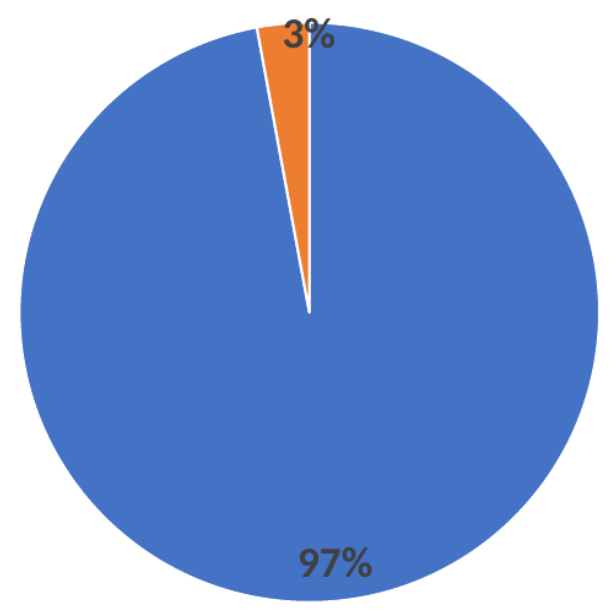

Sí $\square$ No

Figura 5: ¿Ha sido beneficioso para Usted el Diplomado cursado en el DCA?

1.5 Interés en la formación ofrecida por el DCA. Un $88.2 \%$ de los entrevistados expresaron interés en recibir formación del DCA, esto es ocho veces más que quiénes sólo consideraron esta posibilidad. Este resultado coloca al Departamento en condición favorable para ofrecer propuestas de formación nuevas o que retomen, para profundizarlos, algunos de los temas abordados en los diplomados impartidos (ver gráfico de la figura 6).

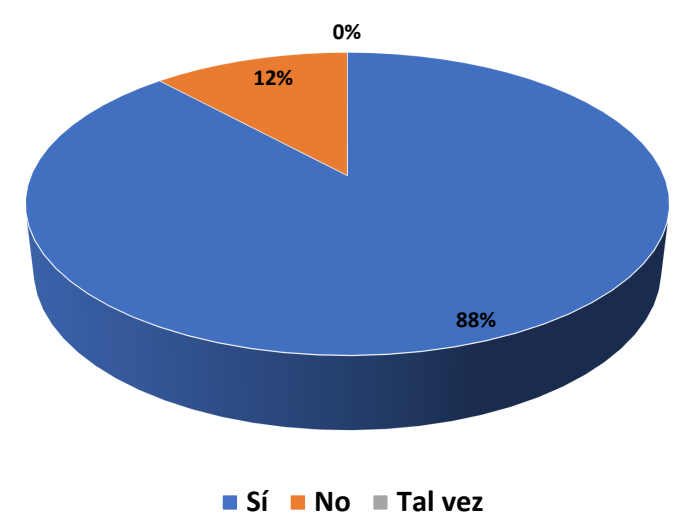

Figura 6: ¿Le interesa cursar un programa formativo con el DCA? 


\section{Resultado de las entrevistas}

2.1 Todos los entrevistados se encuentran trabajando en el rubro aeronáutico en un espectro amplio de empleos que van desde profesores universitarios hasta pilotos incluyendo un consultor en Ciencias Aeronáuticas, dos empleados en temas de capacitación de la AHAC, un gerente de operaciones, un empleado de una OMA, dos miembros de un bufete dedicado a temas aeronáuticos y un propietario de una Centro de Instrucción Aeronáutico.

2.2 Nueve de los diez entrevistados consideran que los conocimientos adquiridos les han sido útiles para el desempeño del propio trabajo y afirman haberlos utilizado para: hacerse una idea de conjunto del campo aeronáutico para conformar una oferta académica, dar a la propia vida profesional una nueva orientación, hacer consultoría en el campo aeronáutico, diferenciar los roles de la aeronáutica civil de los que corresponden a la aeronáutica militar, definir una concepción clara de los asuntos de la aeronáutica civil y mejorar la propia imagen.

2.3 Entre los principales aportes de los Diplomados del DCA se destacan: la comprensión de los perfiles que interactúan en la aeronáutica, la inducción a la investigación en aeronáutica, el fortalecimiento de capacidades para el desempeño de las propias funciones de trabajo, el contacto con la comunidad aeronáutica, el proveer una visión global de las Ciencias Aeronáuticas, el enriquecimiento de los conocimientos sobre aeronáutica, conocimientos para hablar con propiedad de la aeronáutica, el mejoramiento de los servicios ofrecidos, la aplicación de conocimientos para la mejora de procesos, la corrección a terceros que no hacen bien el trabajo que les corresponde, la profundización de conocimientos, la presentación en el aula de situaciones que se habían presentado en la vida cotidiana, la distinción entre la venta de conocimientos y la venta de servicios, una base técnica sólida para el desempeño en las propias funciones, el mejoramiento de la relación con la AHAC, el mejoramiento de la asesoría profesional, buenas relaciones con la comunidad aeronáutica, ver el aeropuerto en su conjunto.

2.4 Nueve de los 10 entrevistados han estado en contacto con el Departamento de Ciencias Aeronáuticas (DCA) y todos dijeron que este Departamento debe seguir impartiendo nuevos Diplomados, se agrega que: no se cuenta con este tipo de oferta en otros lugares con lo cual el DCA está siendo determinante para la aeronáutica más que cualquier otro Departamento de la UNAH.

2.5 A la pregunta sobre si el o los diplomados recibidos les habían motivado para buscar una carrera aeronáutica, cinco entrevistados dijeron que sí, igual número que no; el sí tuvo los siguientes agregados: el Diplomado en Gestión de Sistemas Aeroportuarios motivó a una de las entrevistadas a sacar un Master en el campo aeronáutico, la motivación se dio por el dinamismo y la importancia dada a las clases, dos entrevistados están considerando la posibilidad de un Doctorado. En los casos en los que se dijo que no había una motivación para hacer una carrera aeronáutica, se explicó que esto era por los siguientes motivos: no se ha dado la oportunidad, ya se cuenta con una carrera, la formación en aeronáutica es un instrumento de trabajo solamente para este momento. Seis de los entrevistados están interesados en cursar un posgrado en aeronáutica civil.

2.6 Temas sobre los que el DCA debería hacer nuevos diplomados: manejo de vehículos aéreos no tripulados, SMS, producción Aeronáutica, gestión aeronáutica, aeropuertos eco sostenibles, políticas públicas en materia aeronáutica, seguridad de vuelo, aviación regional, aerodinámica, CRM, sobre legislación aeronáutica, instructores técnicos aeronáuticos, gestión de calidad aeronáutica bajo las normas ISO, derecho aeronáutico, ingeniería aeronáutica, recurrentes para despachadores y personal de tripulación, AVSEC, investigación de accidentes. 


\section{Discusión}

1. Sobre los egresados de Diplomados del DCA.

Dado que estos egresados deben significar para el DCA un medio de comunicación efectiva con la comunidad aeronáutica vale la pena identificar en perspectiva de análisis, el perfil de los egresados, los datos que debería manejar el DCA en relación con esta población y la internacionalización en la oferta de servicios que estos egresados han presentado al propio mercado.

\subsection{Perfil aproximado de ocupación de los egresados.}

Considerando la información obtenida a través las fuentes secundarias y de los instrumentos aplicados se puede inferir que el perfil del egresado de los diplomados hasta ahora desarrollados por el DCA responde a las siguientes características Generales:

- Manejo conceptual: Hasta la fecha el DCA ha desarrollado dos Diplomados en tres ediciones cada uno, el Diplomado en Gestión de Sistemas Aeroportuarios (DGSA) y el Diplomado en Gestión de la Aeronavegabilidad y Mantenimiento Aeronáutico (DGAMA). Estos dos diplomados determinan el dominio conceptual y práctico con el que cuentan los egresados. El DGSA se dio en cinco módulos; el primero denominado Fundamentos de la Aeronáutica Civil abordó los temas de Terminología y principales definiciones utilizadas en la aeronáutica, los antecedentes de la aeronáutica en Honduras, las instancias internaciones y nacionales de la aeronáutica, nociones de sistema aeroportuario, una descripción general de los perfiles técnicos y profesionales en el rubro aeronáutico, las empresas e instituciones del rubro; el segundo módulo, Aeropuertos, operaciones, seguridad y mantenimiento, explicó conceptos como una clasificación de aeropuertos, la seguridad y la higiene en los aeropuertos, la operación y el mantenimiento de un aeródromo; el tercer módulo, administración y finanzas aeroportuarias, propuso las temáticas de los tipos de administraciones que puede tener un aeropuerto, las agencias reguladoras y promotoras de aeropuertos y aeródromos a nivel mundial; las distintas fuentes de financiamiento con las que puede contar un aeropuerto, las dimensiones financiera y operativa del presupuesto de un aeropuerto; el cuarto módulo llamado legislación y desarrollo de servicios aeronáuticos, ofreció los conceptos de la legislación de la aeronáutica civil en Honduras, el desarrollo de servicios aéreos, el mercadeo y la función de las relaciones públicas en un aeropuerto, el emprendedurismo aeroportuario, el impacto económico de un aeropuerto; el quinto y último módulo, llamado planificación aeroportuaria: construcción y sostenibilidad ambiental, desarrolló los temas de la planificación para el mejoramiento de un aeropuerto, el plan maestro aeroportuario, el diseño de aeródromos, la sostenibilidad ambiental aeroportuaria. Por otra parte el DGAMA se desarrolló en cuatro módulos, el primer módulo llamado Estructura organizativa de la aeronavegabilidad y las responsabilidades del Estado, abordó los temas de las responsabilidades del Estado en materia de aeronavegabilidad, la organización del departamento de aeronavegabilidad, el concepto de Certificado Tipo; el segundo módulo, Estado de diseño y estado de fabricación de la aeronave, contó con los temas de la distribución de responsabilidades de los estados de diseño y los estados de fabricación con respecto a los certificados tipo, el proceso de una certificación tipo, los cambios al diseño tipo, la aprobación y certificación de la producción en el mantenimiento de la aeronavegabilidad, los requisitos adicionales cuando el Estado de fabricación no es el Estado de Diseño; el tercer módulo, denominado Certificación de Aeronaves, incluyó los temas requerimiento regulatorio para marcas 
y matrículas de aeronaves, registro y marcas de aeronaves, certificado de homologación de ruidos, certificado de aeronavegabilidad, aprobación de permiso especial de vuelo, certificado de aeronavegabilidad para la exportación; el cuarto y último módulo, Operadores Aéreos Cumplimiento, contó con los siguientes temas: responsabilidad por el mantenimiento de la aeronavegabilidad de las aeronaves, el proceso de certificación de un operador aéreo, el personal gerencial y el sistema de gestión para el mantenimiento de la aeronavegabilidad (control de mantenimiento, planificación, sistema de calidad, almacén de productos aeronáuticos), los requerimientos de instrumentos, equipamiento y equipos de comunicación, el sistema de Manual del operador, las Directivas de Aeronavegabilidad (RAC 39), modificaciones y reparaciones, la información mandatoria de aeronavegabilidad.

- Habilidades. A juzgar por los trabajos de investigación presentados, el desempeño durante el desarrollo de los dos diplomados y los aportes hechos por los egresados en sus ambientes de trabajo se puede afirmar que los egresados muestran las siguientes habilidades:

- Habilidad para aplicar los conocimientos adquiridos a situaciones nuevas,

- Facilidad para presentar proyectos de mejora de procesos,

- Capacidad de adaptación a nuevas funciones dentro del propio trabajo,

- Cumplimiento con instrucciones,

- Capacidad para resolver problemas,

- Aplicación de principios éticos,

- Capacidades para el trabajo en equipo.

1.2 Componentes de un banco de datos del DCA para el manejo de la oferta académica

a. Oferta de educación no formal. No todos los trece proyectos de vinculación en educación no formal, con los que cuenta el Departamento hasta la fecha se encuentran activos y los que registran actividades se encuentran en distintos momentos de su desarrollo, algunos de ellos después de una dos o tres ediciones, no se han vuelto a activar, otros actualmente (agosto 2018) se encuentran en plena actividad, otros están en proceso de conformación y por último, algunos se han presentado en distintas discusiones en el DCA como ideas de proyecto. Esta variedad debería contar con un registro dentro del Departamento de manera que la oferta de académica de vinculación pueda ser planificada por año y período.

b. Oferta de educación formal. Este apartado del banco de datos debería dar seguimiento a las carreras de grado y posgrado del DCA, incluyendo las Carreras que están en proceso de aprobación, las que están en pleno desarrollo, las carreras de las que se está elaborando una investigación diagnóstica y aquellas que ya cuentan con un diagnóstico finalizado. El tema se relaciona con la labor en educación no formal, puesto que con los diplomados impartidos por el DCA, se ha llegado al mercado potencial para las carreras de grado y posgrado que puede ofrecer este Departamento.

c. Egresados de procesos formativos. Conviene al DCA saber de los egresados de todos sus procesos formativos manteniendo actualizada información sobre aspectos como, el número de egresados, perfiles de trabajo en los que se desempeñan, lugares en donde trabajan y necesidades de formación.

d. Proyectos de investigación. Debe existir un archivo con la información básica de las investigaciones, los proyectos de mejora de procesos y otros trabajos relevantes que hayan surgido de 
los diplomados y cursos impartidos por el DCA. Considerando que los estudiantes de Diplomados y los de algunos de los cursos impartidos han preparado documentos con propuestas en algunos casos novedosas y han hecho presentaciones de dichos trabajos, este material sería la materia prima de este apartado del banco de datos.

e. Conferencias, publicaciones y otras actividades académicas del DCA en las que han participado los egresados. Este apartado debería ofrecer información sobre las actividades académicas del Departamento en las que ha contado con la colaboración de egresados de los propios procesos formativos, de hecho hasta ahora los egresados han escrito artículos para la Revista Ciencias Espaciales o han sido coautores, han presentado conferencias y han apoyado el desarrollo de al menos dos de la asesorías que está haciendo el DCA para la comunidad aeronáutica (es el caso de la conformación de una Regulación para el uso de RPAS y el levantamiento de way points para el aeródromo de Utila). A este tipo de colaboraciones se les debería dar seguimiento, pero además a labores similares que atienden los egresados aplicando los conocimientos adquiridos en nuestros procesos formativos o generando nuevos conocimientos a partir de lo aprendido con el DCA.

f. Seguimiento a la publicidad de las actividades de vinculación. Los procesos formativos de vinculación en el DCA, han sido dados a conocer a la comunidad universitaria y, en algunos casos al público en general; en tal sentido es recomendable dar seguimiento a la visibilidad de estos procesos en los distintos medios de comunicación dentro y fuera de la UNAH.

\subsection{Internacionalización de los egresados}

El campo de trabajo de la Aeronáutica es por su naturaleza nacional, regional e internacional; esta característica es notoria en la labor de los egresados de los dos diplomados que han sido objeto de análisis en la presente investigación. Se cuenta con pilotos que desempeñan sus funciones a nivel regional y quien lo hace fuera de la región centroamericana, además se tiene noticia de quienes han realizado una labor de consultoría para empresas e instituciones de fuera de la región centroamericana. El tomar nota de este aspecto debe obligar al DCA a pensar las propias propuestas de formación, dando por hecho que sus egresados deberán prestar sus servicios fuera de las fronteras nacionales y aún más allá de la región centroamericana.

\subsection{Incidencia de egresados en Instituciones y empresas}

El requisito de egreso relacionado con el trabajo que deben presentar los estudiantes de diplomados del DCA ha evolucionado de ser una investigación mínima que puede elaborarse en base a información accesible, a presentarse como una propuesta de mejora de procesos construida con información específica propia de los ambientes en los que se estaban desenvolviendo los alumnos al momento de participar en el diplomado. Se cuenta por ejemplo, con una investigación que subrayó la necesidad de contar en Honduras con un Consejo Aeronáutico Nacional para orientar una política para este campo como ya sucede en Chile, en Ecuador y en República Dominicana; otra investigación examinó el tema de los certificados tipo para aeronaves experimentales y las conclusiones del trabajo sirvieron luego para asesorar a un operador que en Honduras afrontaba problemas con un registro con características similares a las que se dan con este tipo de certificados; otro de los proyectos abordó el tema de la seguridad en un aeródromo municipal logrando recomendaciones útiles e interesantes. Por lo que se refiere a propuestas para la mejora de procesos se han elaborado recomendaciones para el mejoramiento de un hangar que sirve a aeronaves para la fumigación, se han propuesto una 
planificación del manejo de un almacén de repuestos y se ha revisado la atención a las no conformidades para el mantenimiento de una aeronave de un centro de instrucción aeronáutico.

En lo relacionado con la aplicación de conocimientos en la práctica cotidiana se tienen testimonios de un egresado que en fechas recientes (2018) sirvió clases en el primer ejercicio de formación hecho por un centro de instrucción en materia de mantenimiento aeronáutico que fue ofrecido en la Zona Norte de Honduras; pero contó con alumnos del Centro y Sur del País. Este curso se impartió de forma gratuita y aunque no cumpliera con los requisitos para una certificación, con su impartición se cumplió con lo establecido en el Manual de Operativo de una Organización de Mantenimiento Aprobada.

No se debe dejar de mencionar que los conocimientos adquiridos en uno de los Diplomados sirvieron para orientar el trabajo de un bufete que actualmente atiende casos propios del campo aeronáutico. Dos de los egresados que trabajan en este tema consideran que la experiencia del diplomado recibido les permitió pasar de un paradigma de venta de servicios que ofrece intervenciones puntuales a uno de transmisión de conocimientos aplicados en el que se prioriza la resolución de problemas.

Se puede afirmar que uno de los principales aportes de los Diplomados del DCA lo ha recibido la Academia, puesto que los cinco pasos utilizados para los proyectos de investigación y para la conformación de proyectos de mejora de procesos (1. Aspectos generales del proyecto, 2. Metodología, 3. Datos a recolectar, 4 . Resultados a obtener, 5 . Informe del proyecto) ya están siendo aplicados para orientar el trabajo en investigación educativa en uno de los tres grupos conformados dentro de la Comunidad STEM de la UNAH. ${ }^{1}$

\subsection{Incidencia en la oferta académica del DCA}

Hasta la fecha, dado que el DCA no cuenta con Carreras de grado, la labor más relevante del Departamento ha sido la desarrollada en materia de vinculación Universidad Sociedad y, dentro de toda esta oferta, los diplomados representan un lugar central, de hecho la información más significativa que se ha reportado para la elaboración del catálogo de vinculación que se elabora año con año en la FACES proviene de los diplomados; los cuales no solo han generado nuevos temas de investigación, sino que además se han mejorado con la sucesión de ediciones y han logrado que el DCA visualice temas sobre los que es oportuno y necesario generar nuevos procesos formativos en los formatos de educación no formal (cursos, seminarios, conferencias y talleres).

Adicionalmente con los diplomados se han conocido de manera específica una porción significativa de los perfiles profesionales y técnicos que pueblan el campo aeronáutico en Honduras. Cabe mencionar que son o han sido parte del cuerpo de docentes del DCA cuatro de los egresados de los dos diplomados impartidos hasta la fecha y los nombres de los perfiles más prominentes de los

\footnotetext{
${ }^{1}$ Actualmente la Dirección de Innovación Educativa de la UNAH está apoyando la conformación de una Comunidad STEM (Siglas en Ingles que significan Science Technology and Mathematics), la cual se ha distribuido en tres grupos uno para la elaboración de tutoriales para la enseñanza de Matemáticas y temas relacionados, un segundo para la realización de una Feria STEM y un tercero para hacer investigación educativa aplicada a las áreas STEM, este tercer grupo está conformando un Curso denominado IE STEM aplicando los cinco pasos propuestos para los proyectos de investigación e intervención de los diplomados del DCA.
} 
egresados ya son parte del banco de datos de docentes presentados en los planes de estudio de las carreras de grado que al presente están próximas a ser aprobadas en la UNAH.

1.6 Limitación de los impactos logrados.

Los dos diplomados desarrollados por el DCA han sido diseñados cuidando los detalles centrales de todo proceso formativo incluyendo datos relevantes de las empresas e instituciones de procedencia de los participantes, definición clara de los perfiles de egreso de acuerdo a los contenidos establecidos, contenidos pertinentes, una combinación adecuada de teoría y práctica, estrategias pedagógicas adecuadas, uso de plataforma virtual, visitas de campo diseñadas, perfiles adecuados de docentes para cada uno de los módulos de cada diplomado, planificación de recursos pedagógicos y didácticos, asignaciones de tiempos. Toda esta preparación ha garantizado, en alguna medida el éxito relativo de las seis promociones de los diplomados ofrecidos.

Sin embargo, el Departamento es consciente de que los impactos logrados pueden ser mejores si se incorporan aspectos como la intensificación de contactos con empresas e instituciones regionales centroamericanas a las que se pueda ofrecer los diplomados disponibles u otros similares según la demanda de la región. Cabe incluso la posibilidad de incorporar como factor de mejora la conformación de programas conjuntos de formación con universidades más allá de la región centroamericana a nivel continental y aún mundial; en este orden de ideas sería muy provechoso como una prestación extra para los egresados el que el DCA ostentara una Certificación como Centro de Instrucción por parte de OACI. Otro elemento de mejora que está por incorporarse es el establecimiento de convenios de cooperación con empresas del rubro a las cuales se pueda ofrecer formación inicial y recurrente en los campos en los que el Departamento tiene experiencia. Por último y no menos importante para mejorar el impacto positivo de los diplomados impartidos por el DCA, sería cumplir con una de las finalidades de la presente investigación, es decir, establecer las bases para un sistema de seguimiento a egresados en los términos sugeridos en la discusión del presente trabajo, sería un sistema que permitiría la toma de decisiones informadas con respecto a la oferta académica de educación no formal y podría orientar también en alguna medida, la conformación de nuevas carreras de grado y posgrado para el campo de la aeronáutica civil en Honduras y la región centroamericana.

\section{Conclusiones}

1. Una porción importante de los egresados se encuentra activa en el campo aeronáutico y muestran interés por continuar formándose en temas que ellos mismos proponen y que el DCA podría calificar como relevantes y pertinentes para los campos en los que los egresados están empleados.

2. Los conocimientos y habilidades adquiridos o estimulados por los dos diplomados cursados en el DCA, han mejorado el desempeño de los técnicos y profesionales egresados; de los productos de los instrumentos aplicados se percibe cierta destreza en el manejo de habilidades que pueden facilitar en el egresado su adaptación a distintos contextos laborales, entre estas habilidades destacan la aplicación de conocimientos a situaciones nuevas, el asumir nuevas funciones en el propio ámbito laboral, la resolución de problemas y el trabajo en equipo.

3. La cobertura de los espacios de trabajo en donde los egresados prestan sus servicios, representa un abanico amplio de la institucionalidad y el empresariado aeronáutico; dado el perfil de trabajo de los egresados se cuenta con presencia de éstos en: la Autoridad Aeronáutica, Organizaciones de Mantenimiento Aprobadas, líneas aéreas, servicios de ground handlings, centro de instrucción aeronáutica, la Policía Nacional, 
empresas fumigadoras y asesoría legal. Esta presencia le debe significar una ventaja al DCA al momento de promover la propia oferta académica, dando seguimiento a la relación que se ha venido dando con las empresas e instituciones que mantienen activo en Honduras el rubro aeronáutico.

4. Se resalta la importancia de formalizar relaciones con la Autoridad Aeronáutica y con las empresas del rubro aeronáutico para abrir la posibilidad al DCA de ofrecer a estas instancias formación inicial y recurrente, según las capacidades existentes en el Departamento; este relacionamiento puede iniciar con una colaboración planificada en conjunto para luego establecerse en forma de convenios.

5. Considerando que los egresados tienen una percepción positiva de la formación recibida, la cual es considerada útil puesto que ha contribuido a la mejoría del propio desempeño y que además la mayoría de los entrevistados consideran que el DCA debe seguir formando en distintos temas de la aeronáutica; se puede afirmar que nuevos programas formativos continuarán teniendo demanda, siempre que se renueven las temáticas ofrecidas (los entrevistados enlistaron 18 nuevas temáticas a abordar) y se aproveche el momento favorable que se está dando para el DCA en el contexto nacional de formación para la comunidad aeronáutica.

6. Una característica que debe asignársele a la oferta académica es su orientación hacia estudiantes que cumplan con una o más de estos requisitos: secundaria finalizada, que se encuentren estudiando en el nivel superior, empleados en el rubro aeronáutico con título de grado universitario.

7. De acuerdo con las actividades cotidianas de los propios campos de trabajo de los egresados se constata interés por las siguientes áreas de formación:

a. Temas de interés general: Manejo de Vehículos aéreos no tripulados, Sistemas de seguridad operativa (SMS), Seguridad de la aviación (AVSEC), aeropuertos eco sostenibles, Seguridad de Vuelo, Aviación Regional.

b. Temas legales y administrativos: Gestión Aeronáutica, CRM, Producción Aeronáutica, sobre Legislación aeronáutica, Gestión de calidad aeronáutica bajo las normas ISO.

c. Ecología y políticas públicas: Aeropuertos eco sostenibles, Políticas públicas en materia aeronáutica.

d. Formación científica e instrucción: Aerodinámica, Instructores Técnicos Aeronáuticos, Ingeniería Aeronáutica, recurrentes para despachadores y personal de tripulación, investigación de accidentes.

\section{Listado de siglas utilizadas}

AD Notes: Directivas de aeronavegabilildad (en inglés: Airworthiness Directives Notes).

AVSEC: Aviation Security (Seguridad de la Aviación).

CRM: Crew Resource Management (Gestión del Personal de Cabina).

DCA: Departamento de Ciencias Aeronáuticas.

DGAMA: Diplomado en Gestión de la Aeronavegabilidad y Mantenimiento Aeronáutico.

DGSA: Diplomado en Gestión de Sistemas Aeroportuarios.

ISO: International Standard Organization (Organización Internacional de Estándares).

OACI: Organización de Aviación Civil Internacional.

RAC: Regulación de Aeronáutica Civil.

SMS: Safety Managment System (Sistema de Gestión de la Seguridad). 
STEM: Science Technology, Engineering, Mathematics (Ciencia, Tecnología, Ingeniería, Matemáticas).

UNAH: Universidad Nacional Autónoma de Honduras.

\section{Referencias}

DCA (2015). Informe final diplomado en gestión de sistemas aeroportuarios. Tegucigalpa: archivo del Departamento de Ciencias Aeronáuticas de la Facultad de Ciencias Espaciales, UNAH.

DCA (2018a). Informe final curso formación de instructores técnicos aeronáuticos. Tegucigalpa: archivo del Departamento de Ciencias Aeronáuticas de la Facultad de Ciencias Espaciales, UNAH.

DCA (2018b). Informe final diplomado en gestión de la aeronavegabilidad y mantenimiento aeronáutico. Tegucigalpa: archivo del Departamento de Ciencias Aeronáuticas de la Facultad de Ciencias Espaciales, UNAH.

DCA (2018c). Navegación aérea, tendencias internacionales y su influencia en la región centroamericana. Tegucigalpa: archivo del Departamento de Ciencias Aeronáuticas de la Facultad de Ciencias Espaciales, UNAH.

del Moral Bueno, A., Pazos Sierra, J., Rodriguez Fernandez, E., Rodriguez-Paton Aradas, A., y Suarez Garaboa, S. (2007). Gestión del conocimiento. Editorial: Paraninfo.

DVUS (2016). Informe del primer estudio de seguimiento a graduado. Dirección de Vinculación Universidad Sociedad. Tegucigalpa: Editorial Universitaria.

UNAH (2008). Reglamento de vinculación universidad-sociedad. Tegucigalpa: La Gaceta.

UNAH (2009). Modelo educativo. Tegucigalpa : Editorial Universitaria.

UNAH (2015). Normas académicas. Tegucigalpa : Editorial Universitaria. 